\title{
Immunologic response of mRNA SARS-CoV-2 vaccination in adolescent kidney transplant recipients
}

\author{
Clarkson Crane $^{1}\left[\right.$ [D $\cdot$ Erin Phebus $^{2} \cdot$ Elizabeth Ingulli $^{1}[$
}

Received: 25 June 2021 / Revised: 25 July 2021 / Accepted: 26 July 2021 / Published online: 15 September 2021

(c) IPNA 2021

\begin{abstract}
Background In the general population, mRNA SARS-CoV-2 vaccines are highly efficacious. Early reports suggest a diminished antibody response in immunosuppressed adult solid organ transplant (SOT) patients, but this has not been reported in pediatrics.

Methods Adolescent kidney transplant recipients (KTR) at our center who received both doses of an mRNA SARS-CoV-2 vaccine had SARS-CoV-2 spike (S) protein antibody presence evaluated 4-8 weeks after their second dose of the vaccine as part of routine clinical care.

Results Thirteen of 25 fully vaccinated patients $(52 \%)$ had a positive spike antibody. Median age of participants was 19 years old (IQR 18-20) and the median time from transplant was 5 years (IQR 4-9 years). KTR were treated with an immunosuppression regimen including a calcineurin inhibitor, corticosteroid, and antimetabolite (9 with mycophenolate, 3 with azathioprine, and 1 without an antimetabolite due to viremia). Of those who had an antibody response, fewer had a mycophenolate-containing immunosuppressant regimen than non-responders. There was a trend toward better vaccine response and higher anti-S antibody titers at lower doses of mycophenolate. Three patients with prior COVID-19 infection all had a positive antibody response.

Conclusion Our results suggest vaccine response in adolescent KRT is lower than that of the general population, but similar to that previously described in adult SOT patients and slightly better than that seen in adult KTR. This data demonstrates vaccination is safe and supports immunizing KTR who remain hesitant. Future studies should focus on better understanding of the cellular immune response to vaccination and strategies to enhance vaccine immunogenicity in pediatric SOT patients.
\end{abstract}

Keywords SARS-CoV-2 $\cdot$ COVID-19 $\cdot$ mRNA vaccine $\cdot$ Pediatric $\cdot$ Adolescent $\cdot$ Kidney transplant

\section{Introduction}

Coronavirus disease 2019 (COVID-19) is caused by severe acute respiratory syndrome coronoavirus-2 (SARS-CoV-2) and has a high rate of complications and mortality in kidney and other solid organ transplant (SOT) recipients [1,2]. There is a paucity of literature focused on immunocompromised pediatric and adolescent patients and their potential

Clarkson Crane

crcrane@health.ucsd.edu

1 Department of Pediatrics, Division of Pediatric Nephrology, University of California At San Diego and Rady Children's Hospital, 3020 Children's Way MC 5173, San Diego, CA 92123, USA

2 Kidney Transplant Program, Rady Children's Hospital, San Diego, CA, USA risk of severe COVID-19 disease. Phase 3 studies of mRNA SARS-CoV-2 vaccines showed excellent vaccine efficacy in healthy participants; however, immunosuppressed pediatric participants were not enrolled [3, 4].

There is evidence to suggest the humoral immune response to various vaccines may be blunted in solid organ transplant recipients [5]. Specifically, in pediatric kidney transplant recipients (KTR) receiving the inactivated influenza vaccine, decreased seroconversion for influenza A compared to healthy controls has been observed [6]. Cellular response to vaccination in SOT patients is less well-characterized. However, it has been shown that SOT recipients are able to mount a T-cell response to influenza vaccination, although it is much stronger in those who were naturally infected [7].

Recent reports also suggest a diminished antibody response to the mRNA SARS-CoV-2 vaccine in adult 
kidney and other SOT recipients, particularly those with an immunosuppression regimen that contains an antimetabolite [8-11]. To our knowledge, the humoral response of adolescent KTR to SARS-CoV-2 has not been reported. The present study quantifies the proportion of adolescent and pediatric patients who developed a humoral response to the SARS-CoV-2 S (spike) antigen at our center. Similar to what has been reported in adult studies, we hypothesize there will be lower rates of antibody response in KTR receiving a mycophenolate-based immunosuppression regimen.

\section{Methods}

\section{Study population}

The prospective and retrospective kidney transplant database at Rady Children's Hospital, San Diego, was utilized to identify eligible patients. When permitted by local guidelines, KTR who were over 16 years old and greater than 6 months since their transplant were notified of vaccine eligibility and encouraged to schedule an appointment. KTR were excluded if they did not receive both doses of the vaccine, were within 6 months of transplant, or received blood products within the past 6 months.

\section{Data}

Data were extracted from electronic health records at Rady Children's Hospital San Diego. Variables obtained included age, sex, time from transplant, prior COVID-19 infection or hospitalization, prior SARS CoV-2 nucleocapsid (N) antibody presence, and type and dose of antimetabolite at time of vaccination. SARS-CoV-2 antibody assays were obtained as part of routine follow-up appointments a minimum of 4 weeks after the final vaccine dose. We utilized the Abbott chemiluminescent microparticle immunoassay or Siemens Atellica IM SARS-CoV-2 IgG. Both assays are commercially available and were designed to be specific to the receptor binding domain of the $\mathrm{S} 1$ subunit of the IM SARS-CoV-2 spike protein. Patients were characterized as vaccine responders if they had an antibody titer greater than $50 \mathrm{AU} / \mathrm{mL}$ for the Abbott assay or greater than 1.0 index for Siemens and were considered non-responders if below these thresholds. Two different assays were used because our center's lab transitioned from sending out SARS-CoV-2 IgG studies to performing in-house testing during our period of data collection.

\section{Outcome}

The primary outcome was the proportion of patients who were SARS-CoV-2 spike-protein IgG seropositive.
Additional outcomes included evidence of prior infection (either symptoms in the setting of a positive PCR test or SARS-CoV-2 N IgG seropositivity) and association of type and dose of antimetabolite with antibody response.

\section{Statistical analysis}

Continuous variables are reported as mean and standard deviations (SD) or as median and interquartile range (IQR). Categorical variables are described as absolute numbers and percentages. For categorical variables, the chi-square statistic was used to assess the statistical significance between groups. Differences between antibody levels and antimetabolite dosing in both groups were compared by the Mann-Whitney $U$ test. Two-sided $P<0.05$ was considered to be statistically significant. Statistical analysis was performed with IBM SSPS v27.

\section{Ethics}

The institutional review boards at the University of California, San Diego and Rady Children's Hospital, San Diego approved this study as part of the center's Retrospective and Prospective Kidney Transplant Database.

\section{Results}

Of 40 vaccine-eligible KTR in our program, 30 received two doses of a SARS-CoV-2 mRNA vaccine. One patient received the mRNA-1273 (Moderna) vaccine and 29 patients received BNT162b2 (Pfizer-BioNTech). Of these patients, 25 (83\%) had anti-S antibody titers obtained and were included for analysis. The median age was 19 years old (IQR 18-20), 11 (44\%) were female, and the median time from transplant was 5 years (IQR 4-9 years). Four patients (16\%) were treated for rejection within 6 months prior to lab draw (one acute T-cell mediated rejection and three chronic antibody-mediated rejection). Three patients (12\%) had prior symptomatic COVID-19 infections and developed antibodies to SARS-CoV-2 N protein.

Antibody titers were checked a median of 45 days (IQR 31-56 days) following the second dose of the vaccine. Thirteen patients $(52 \%)$ had detectable antibody titer and were considered responders. Demographics of responders and non-responders can be seen in Table 1. All three patients with prior COVID-19 infection were positive for anti-S antibody. The three KTR treated for chronic antibody-mediated rejection (one with IVIG and tocilizumab, one with IVIG alone, and one with tocilizumab alone) did not develop anti-S antibodies. One KTR treated for acute T-cell mediated rejection with a steroid pulse alone developed anti-S antibodies, although she also had a history of confirmed 
Table 1 Comparison of KTR with positive vs negative SARS CoV-2 spike antibody.

SARS CoV-2 spike antibody

\begin{tabular}{llll}
\hline Variable & Positive & Negative & p value \\
\hline Number (\%) & $13(52)$ & $12(48)$ & \\
Sex, female (\%) & $4(31)$ & $7(58)$ & 0.17 \\
Years post-transplant (SD) & $6.6(5.4)$ & $7.7(5.4)$ & 0.54 \\
Treatment for rejection within & $1(8)$ & $3(25)$ & 0.24 \\
$\quad$ 6 months (\%) & & & \\
Prior COVID-19 infection (\%) & $3(23)$ & 0 & 0.08 \\
Number of KTR taking MMF (\%) & $9(69)$ & $12(100)$ & 0.04 \\
$\begin{array}{l}\text { MMF dose mg/m2/day (SD) } \\
\text { Number of KTR taking Azathioprine }\end{array}$ & $3(23)$ & 0 & 0.06 \\
$\quad$ (\%) & & & \\
\hline
\end{tabular}

$M M F$, mycophenolate mofetil; $S D$, standard deviation

COVID-19 infection. One KTR receiving IVIG for autoimmune disease did not develop anti-S antibodies. No patients were treated with B-cell depleting agents within the year prior to vaccination.

Of vaccine responders, all had an immunosuppression regimen including an antimetabolite, except for one in whom the antimetabolite was held due to EBV viremia. Three patients in this group were treated with azathioprine (average dose $1.75 \mathrm{mg} / \mathrm{kg}$ ) and the remaining 9 were receiving mycophenolate with a mean dose of 719 (SD 73) $\mathrm{mg} / \mathrm{m}^{2} /$ day.

All vaccine non-responders were receiving treatment with mycophenolate-based immunosuppression. The mean dose was $750(\mathrm{SD} 114) \mathrm{mg} / \mathrm{m}^{2} /$ day. While this was higher than that of vaccine responders, it did not reach statistical significance $(P=0.55)$.

Quantitative anti-S antibody titers were measured for the 20 patients in whom the Abbott assay was obtained. The median titer for responders was 2067 (IQR 159-8314) AU/ $\mathrm{mL}$. Those taking azathioprine had a higher average titer than those taking mycophenolate at $6627 \mathrm{AU} / \mathrm{mL}$ vs. 2985 $\mathrm{AU} / \mathrm{mL}(P=0.12)$.

The vaccine was well tolerated by all patients in our cohort and there were no serious adverse events or hospitalizations related to vaccination. There were no rejection episodes subsequent to vaccination. Additionally, there have been no reported cases of COVID-19 infection in our study population since the start of the vaccination effort.

\section{Discussion}

Our results suggest immunogenicity to the SARS-CoV-2 mRNA vaccines in adolescent KTR is lower than that observed in the general population [12]. Overall, $52 \%$ in our cohort had detectable anti-S protein antibodies. COVID-19 infection and the emergence of SARS-CoV-2 variants pose a substantial threat to immunocompromised hosts, as KTR are at high risk for complications [1].

Our 52\% response rate is similar to that described in adult SOT patients and slightly better than that reported in adult KTR. Boyarsky et al. published one of the initial reports of antibody response in SOT patients following dose 1 and dose 2 of a SARS-CoV-2 mRNA vaccine. Of the 658 participants, 357 (54\%) responded to the vaccine after a second dose [8]. Similarly, Marinaki et al. described a 59\% rate of antibody detection in a cohort of 34 adult SOT patients [9]. In contrast, Grupper et al. reported $38 \%$ of 136 KTR had a positive serology after the two-dose series and there was a significantly higher antibody titer in non-immunosuppressed controls [10]. Rozen-Zvi et al. demonstrated positive S antibodies in 112 of 308 (36\%) of adult KTR [11]. The younger age of our participants may explain the better response seen in our adolescent KTR versus the responses of adult KTR in the literature.

Both studies of KTR noted a trend toward improved antibody response in younger patients and those with less mycophenolate exposure $[10,11]$. As this is consistent with our findings, it is likely that increased immunogenicity and distinct pharmacokinetics seen in pediatric patients explain the better rate of antibody response seen in younger patients. Additionally, Mycophenolate mofetil (MMF) has been shown to decrease humoral immunity and antibody production [13]. Similar to reports in adults, our study showed a trend toward decreased response with use of MMF at higher doses and a significantly higher number of non-responders were treated with MMF versus responders. This is also consistent with prior work showing decreased influenza vaccine immunogenicity in pediatric KTR with more MMF exposure [6]. Alteration of immunosuppression regimens to decrease MMF dose or altogether hold MMF at the time of vaccination is one possible solution to enhance humoral immunity. This is controversial, as there is no strong evidence to guide development of these protocols. Until there is more data associating less MMF with better anti-SARS-CoV-2 response, we would advise caution in altering immune suppression regimens for the sole purpose of immunization, as this could result in unintended consequences such as increased risk of acute rejection or donor-specific antibody formation.

It is important to acknowledge the limitations of only measuring antibody levels as a vaccine response, as it does not measure T-cell immunity. Cellular immunity is likely not as impacted by MMF and could confer partial protection against COVID-19 infection. T-cell-specific responses to mRNA vaccination in SOT patients are currently being studied, and preliminary work appears to suggest a cellular response may be more robust than a humoral response in this patient population and may confer some protection [14]. 
Observations at our center are consistent with these findings, as there have been no documented cases of COVID-19 infection in KTR since vaccination efforts began. In contrast, 12 of $84(14 \%)$ KTR followed at our center developed symptomatic infection before the mRNA vaccines were widely available.

While the amount of adult literature on this topic is expanding, there is little information available about vaccine response in immunosuppressed pediatric KTR. Results from our center's adolescent population can help contribute toward this gap of knowledge. Although limited by small sample size, the primary strength of our study is its novelty showing the SARS-CoV-2 mRNA vaccine is well tolerated and partially effective in a population of adolescent KTR. As evidenced by no serious adverse events or episodes of rejection, vaccination is a safe intervention in this population. These findings support continued efforts to encourage immunization of eligible KTR who remain hesitant.

Vaccine hesitancy is an important barrier to SARS-CoV-2 immunization in SOT patients. Among the entire vaccine eligible population in our study, seven patients $(17.5 \%)$ were hesitant. While SARS-CoC-2 vaccine acceptance rates are not well-characterized in the SOT population, this appears to be better than a $34 \%$ hesitancy rate described in KTR in New York City and consistent with a survey of SOT patients in which $87 \%$ expressed willingness to get vaccinated if recommended by a transplant provider $[15,16]$. We attribute our relatively low vaccine hesitancy rate to trusting providerfamily relationships, emphasizing vaccine safety, mandating routine vaccinations prior to transplant and referencing recommendations from trusted providers, professional societies, and patient/caregiver support groups. Additionally, our center will not transplant patients unless they are fully vaccinated, so the higher rate of SARS-CoV-2 vaccine acceptance may reflect preselecting a patient population more amenable to receiving vaccinations. Our early experience with vaccinating younger patients (12 to 16 years old) suggests hesitancy may be more prevalent and novel means to encourage vaccination of all eligible patients may be needed.

Various strategies to optimize anti-S humoral response in adult SOT patients have been proposed. Providing a third "booster" vaccine dose in immunosuppressed patients suggests promising preliminary results [17]. As vaccine eligibility expands to younger patients, future studies should focus on similar strategies to optimize immunogenicity and better characterize the cellular response to mRNA vaccination in pediatric KTR.

Acknowledgements Study data were collected and managed using REDCap electronic data capture tools hosted at the University of California, San Diego (CTRI grant support UL1TR001442). We also would like to thank kidney transplant personnel Kristin Zeeb MA, Claudia Avila MA, Courtney Benavides RN, and Cheryl Ripp PNP for their assistance with patient coordination and lab draws.
Author contribution All authors contributed to the study conception and design. Material preparation, data collection, and analysis were performed by CC and EP. Data interpretation was performed by $\mathrm{CC}$ and EI. The first draft of the manuscript was written by CC. EI commented on subsequent versions of the manuscript. EI supervised the study. All authors read and approved the final manuscript.

Data availability The datasets generated during and/or analyzed during the current study are available from the corresponding author on reasonable request.

\section{Declarations}

Ethics approval The Retrospective and Prospective Kidney Transplant Database involving human participants were in accordance with the ethical standards of the institutional and national research committee and with the 1964 Helsinki Declaration and its later amendments or comparable ethical standards. The Human Investigation Committee (IRB) of the University of California San Diego and Rady Children's Hospital approved this study.

Consent to participate The need for consent was waived by IRB due to minimal risk to participants.

Consent for publication The need for consent was waived by IRB due to minimal risk to participants.

Conflict of interest The authors declare no conflict of interest.

\section{References}

1. Cravedi P, Mothi SS, Azzi Y, Haverly M, Farouk SS, Pérez-Sáez MJ et al (2020) COVID-19 and kidney transplantation: results from the TANGO International Transplant Consortium. Am J Transplant 20:3140-3148. https://doi.org/10.1111/ajt.16185

2. Caillard S, Anglicheau D, Matignon M, Durrbach A, Greze C, Frimat L et al (2020) An initial report from the French SOT COVID Registry suggests high mortality due to COVID-19 in recipients of kidney transplants. Kidney Int 98:1549-1558. https://doi.org/ 10.1016/j.kint.2020.08.005

3. Polack FP, Thomas SJ, Kitchin N, Absalon J, Gurtman A, Lockhart S et al (2020) Safety and efficacy of the BNT162b2 mRNA Covid-19 vaccine. N Engl J Med 383:2603-2615. https://doi.org/ 10.1056/NEJMoa2034577

4. Baden LR, El Sahly HM, Essink B, Kotloff K, Frey S, Novak R et al (2021) Efficacy and safety of the mRNA-1273 SARS-CoV-2 vaccine. N Engl J Med 384:403-416. https://doi.org/10.1056/ NEJMoa2035389

5. Eckerle I, Rosenberger KD, Zwahlen M, Junghanss T (2013) Serologic vaccination response after solid organ transplantation: a systematic review. PLoS ONE 8:e56974. https://doi.org/10.1371/ journal.pone.0056974

6. Nailescu C, Xu X, Zhou H, Hall H, Wilson AC, Leiser JD et al (2011) Influenza vaccine after pediatric kidney transplant: a Midwest Pediatric Nephrology Consortium study. Pediatr Nephrol 26:459-467. https://doi.org/10.1007/s00467-010-1729-1

7. L'Huillier AG, Ferreira VH, Hirzel C, Nellimarla S, Ku T, Natori $Y$ et al (2020) T-cell responses following natural influenza infection or vaccination in solid organ transplant recipients. Sci Rep 10:10104. https://doi.org/10.1038/s41598-020-67172-6

8. Boyarsky BJ, Werbel WA, Avery RK, Tobian A, Massie AB, Segev DL, Garonzik-Wang JM (2021) Antibody response to 
2-dose SARS-CoV-2 mRNA vaccine series in solid organ transplant recipients. JAMA 325:2204-2206. https://doi.org/10.1001/ jama.2021.7489

9. Marinaki S, Adamopoulos S, Degiannis D, Roussos S, Pavlopoulou ID, Hatzakis A, Boletis IN (2021) Immunogenicity of SARSCoV-2 BNT162b2 vaccine in solid organ transplant recipients. Am J Transplant 21:2913-2915. https://doi.org/10.1111/ajt.16607

10. Grupper A, Rabinowich L, Schwartz D, Schwartz IF, Ben-Yehoyada $\mathrm{M}$ et al (2021) Reduced humoral response to mRNA SARSCoV-2 BNT162b2 vaccine in kidney transplant recipients without prior exposure to the virus. Am J Transplant 21:2719-2726. https://doi.org/10.1111/ajt.16615

11. Rozen-Zvi B, Yahav D, Agur T, Zingerman B, Ben-Zvi H, Atamna A et al (2021) Antibody response to SARS-CoV-2 mRNA vaccine among kidney transplant recipients: a prospective cohort study. Clin Microbiol Infect 27:1173.e1-1173.e4. https://doi.org/10. 1016/j.cmi.2021.04.028

12. Walsh EE, Frenck RW Jr, Falsey AR, Kitchin N, Absalon J, Gurtman A et al (2020) Safety and immunogenicity of two RNA-based Covid-19 vaccine candidates. N Engl J Med 383:2439-2450. https://doi.org/10.1056/NEJMoa2027906N

13. Rentenaar RJ, van Diepen FN, Meijer RT, Surachno S, Wilmink $\mathrm{JM}$ et al (2002) Immune responsiveness in renal transplant recipients: mycophenolic acid severely depresses humoral immunity in vivo. Kidney Int 62:319-328. https://doi.org/10.1046/j.15231755.2002.00425.x
14. Bertrand D, Hamzaoui M, Lemée V, Lamulle J, Hanoy M, Laurent C et al (2021) Antibody and T cell response to SARS-CoV-2 messenger RNA BNT162b2 vaccine in kidney transplant recipients and hemodialysis patients. J Am Soc Nephrol. https://doi.org/10. 1681/ASN.2021040480J

15. Ou MT, Boyarsky BJ, Zeiser LB, Po-Yu Chiang T, Ruddy J, Van Pilsum RSE et al (2021) Kidney transplant recipient attitudes toward a SARS-CoV-2 vaccine. Transplant Direct 7:e713. https:// doi.org/10.1097/TXD.0000000000001171

16. Tsapepas D, Husain SA, King KL, Burgos Y, Cohen DJ, Mohan S (2021) Perspectives on COVID-19 vaccination among kidney and pancreas transplant recipients living in New York City. Am J Health Syst Pharm. https://doi.org/10.1093/ajhp/zxab272

17. Werbel WA, Boyarsky BJ, Ou MT, Massie AB, Tobian A, Garonzik-Wang JM, Segev D (2021) Safety and immunogenicity of a third dose of SARS-CoV-2 vaccine in solid organ transplant recipients: a case series. Ann Intern Med. https://doi.org/10.7326/ L21-0282

Publisher's note Springer Nature remains neutral with regard to jurisdictional claims in published maps and institutional affiliations. 